\title{
Premature atherosclerosis in primary antiphospholipid syndrome: preliminary data
}

\author{
P R J Ames, A Margarita, K B Sokoll, M Weston, V Brancaccio
}

Objective: To investigate the atherosclerosis hypothesis in primary antiphospholipid syndrome (PAPS).

Methods: The intima media thickness (IMT) of carotid arteries and other cardiovascular risk factors was measured in 20 patients with PAPS (mean (SD) age 35 (12) years) and in 20 controls matched for age and sex (34 (12) years).

Results: The frequency of smoking, hypertension, and dyslipidaemia was similar in the two groups, but plasma homocysteine was higher in patients with PAPS (mean (SD) 11.9 (6.2) v 8.2 (3.4) $\mu \mathrm{mol} / \mathrm{l}, p=0.037)$. The IMT was slightly greater in patients with PAPS than in controls at the carotid bifurcation (mean (SD) $0.61 \quad(0.24) \vee 0.48$ $(0.09) \mathrm{mm}, \mathrm{p}=0.04)$ and internal carotid artery $(0.52$ $(0.22) \vee 0.40(0.08), p=0.01)$. These differences were more evident in patients aged $>40$ years than in those aged $<30$ years at the carotid bifurcation $(0.76(0.25) \vee 0.55$ (0.06), $\mathrm{p}=0.0007)$ and internal carotid artery $(0.63(0.25) \mathrm{v}$ $0.45(0.09), p=0.02)$; no differences were seen in the younger age group compared with controls.

Conclusion: Atherosclerosis is a possibility in patients with PAPS in their fourth decade of life or older.

$\mathrm{T}$ he thrombotic tendency of the antiphospholipid syndrome (APS) shares several pathways with atherosclerosis. ${ }^{1}$ Antiphospholipid antibody (aPL) related atherosclerosis has been described in systemic lupus erythematosus, ${ }^{2-4}$ and two studies have discussed the presence of atherosclerosis in primary APS (PAPS). ${ }^{5}{ }^{6}$ In addition to the association of aPL with autoimmune disease, a higher prevalence of aPL has been detected in young patients with objectively documented peripheral vascular disease. ${ }^{78}$ We have previously shown that IgG anticardiolipin (aCL) titre independently predicts the intima media thickness (IMT) of carotid arteries, a surrogate marker of atherosclerosis, in patients with PAPS, ${ }^{9}$ and in this report we evaluate whether premature atherosclerosis is a feature of our patients.

\section{PATIENTS AND METHODS Patients}

We investigated 20 patients with PAPS who had been followed up for a mean (SD) of 4 (1.4) years. They were evaluated for a thrombotic event and found to be positive for lupus anticoagulant (LA) and aCL on two separate occasions 6 weeks apart. They all met the Sapporo criteria for APS. ${ }^{10}$ To keep the focus on vascular disease, women who had had miscarriages were not included in this cohort. Magnetic resonance imaging, Doppler ultrasound, and electrocardiography were used to diagnose thrombosis according to the vascular bed affected. An equal number of healthy control subjects of similar age and sex matched the patients with
PAPS. Table 1 presents the demographics and clinical features of patients and controls.

\section{Methods}

The presence of LA was determined from the activated partial thromboplastin time, dilute Russell viper venom time, and kaolin clotting time. After detecting an inhibitor by mixing studies, the platelet neutralisation procedure in the activated partial thromboplastin time and dilute Russell viper venom time determinations was used to confirm the presence of an LA in accordance with published guidelines. ${ }^{11}$ Serum IgG aCL were measured by an enzyme linked immunosorbent assay (ELISA; Cambridge Life Sciences, UK). A normal range was determined from 60 healthy hospital personnel with a cut off point of $5 \mathrm{GPL} \mathrm{U} / \mathrm{ml}$, which is five standard errors above the geometric mean (4.1; SEM 0.2; 95\% confidence interval 3.7 to $4.5)$. Titres $<20$ GPL were defined as low positive, between 21 and 80 GPL medium positive, and $>81$ GPL high positive.

Plasma homocysteine (HC) was measured by an ELISA (Bio-Rad, Oslo, Norway) as previously described. ${ }^{9}$ The upper limit of normal HC (mean+3SD) was $13.3 \mu \mathrm{mol} / \mathrm{l}$. This was calculated from 36 healthy subjects ( 10 male, 26 female) who were not homozygous for the methylentetrahydrofolate reductase $\mathrm{C}^{677 \rightarrow \mathrm{T}}$ mutation. The intra- and interassay coefficients of variability were $6.6 \%$ and $7.5 \%$, respectively. Cholesterol, high density lipoprotein, low density lipoprotein, and triglycerides were laboratory grade measurements.

\section{IMT measurement}

An experienced angiologist (AM) performed a detailed ultrasound examination of the carotid arteries in patients with PAPS and healthy controls by an Aloka 2000 sonograph equipped with a 5-10 MHz linear transducer. Image acquisition followed the Atherosclerosis Risk in Communities Study protocol. ${ }^{12}$ Transverse and longitudinal views of the far wall of the right and left common carotid arteries ( $10 \mathrm{~mm}$ distal to the carotid bifurcation), right and left carotid bifurcation, and internal carotid arteries were taken with the patient in the supine position with the neck slightly extended. The means of three measurements taken $1 \mathrm{~cm}$ apart at the three different sites were computed for each region. Right and left measurements were averaged for the statistical analysis. Intrareader reproducibility was assessed in two ways. Firstly, the same carotid measurement was performed twice on 20 subjects, yielding a coefficient of agreement of almost $97 \%$. Secondly, the same IMT measurements were performed six times on one subject over a 2 month period, yielding a coefficient of variability of between 3 and $4 \%$ according to the carotid segment under study. The study was carried out with

Abbreviations: aCL, anticardiolipin antibodies; aPL, antiphospholipid antibodies; ELISA, enzyme linked immunosorbent assay; HC, homocysteine; IMT, intima media thickness; LA, lupus anticoagulant; PAPS, primary antiphospholipid syndrome 


\begin{tabular}{|c|c|c|}
\hline & CTR & PAPS \\
\hline Participants (No) & 20 & 20 \\
\hline Female/male & $13 / 7$ & $13 / 7$ \\
\hline Age (years), mean (SD) & $34(12)$ & $35(12)$ \\
\hline $\operatorname{lgG} \mathrm{aCL}$ & & \\
\hline$<20$ & 0 & $2^{*}$ \\
\hline $20-80$ & 0 & 3 \\
\hline$>80$ & 0 & \\
\hline IgG aCL (GPL), mean (SD) & & $177(134)$ \\
\hline Lupus anticoagulant & 0 & 20 \\
\hline Platelets $<50 \times 10^{9} / \mathrm{I}$ & 0 & 3 \\
\hline IS & 0 & 4 \\
\hline Ml & 0 & 1 \\
\hline DVT & 0 & 11 \\
\hline $\mathrm{DVT}+\mathrm{MI}$ & 0 & 1 \\
\hline PE & 0 & 3 \\
\hline Smoking & 4 & 6 \\
\hline Hypertension & 1 & 1 \\
\hline Diabetes & 0 & 0 \\
\hline Obesity & 0 & 0 \\
\hline High cholesterol & 1 & 1 \\
\hline High triglycerides & 0 & 3 \\
\hline High homocysteine & 3 & $8 \dagger$ \\
\hline Warfarin & 0 & 20 \\
\hline \multicolumn{3}{|c|}{$\begin{array}{l}\text { The number of patients is shown unless otherwise stated. } \\
\text { CTR, controls; PAPS, primary antiphospholipid syndrome, IS, ischaemic } \\
\text { stroke; MI, myocardial infarction; DVT, deep vein thrombosis; PE, } \\
\text { pulmonary embolism; } \\
\text { "Both patients had a lupus anticoagulant; †NS by Fisher's exact test. }\end{array}$} \\
\hline
\end{tabular}

ethics approval and all participants gave written consent before entering the study.

\section{Statistics}

Statistical analysis was carried out with the Prism statistical package. Mann-Whitney and Fisher's exact test were used to compare distribution and frequencies between groups, respectively.

\section{RESULTS}

Most patients (18/20 (90\%)) had medium to high positive serum IgG aCL (table 1). The frequency of smoking, obesity, hypertension, diabetes, dyslipidaemia, and plasma HC above the upper limit of normal $(13 \mu \mathrm{mol} / \mathrm{l})$ was similar between patients and controls, although mean (SD) plasma HC

Table 2 Intima media thickness in study groups

\begin{tabular}{llll}
\hline & CTR $(\mathbf{n}=20)$ & PAPS $(\mathbf{n}=20)$ & $p$ Value \\
\hline IMT $(\mathrm{mm})$ overall & & & \\
CC & $0.43(0.08)$ & $0.53(0.28)$ & 0.18 \\
B & $0.48(0.09)$ & $0.61(0.24)$ & 0.04 \\
IC & $0.40(0.08)$ & $0.52(0.22)$ & 0.01 \\
Disease duration (years) & & $7(4)$ & \\
& & & \\
IMT (mm) $<31$ years & CTR (n=10) & PAPS $(\mathbf{n = 1 0 )}$ & \\
CC & $36(0.05)$ & $0.40(0.09)$ & 0.31 \\
B & $41(0.04)$ & $0.45(0.08)$ & 0.30 \\
IC & $34(0.04)$ & $0.41(0.12)$ & 0.13 \\
Disease duration (years) & & $5(3)$ & \\
& & & \\
IMT (mm) $>40$ years & & & \\
CC & $0.49(0.04)$ & $0.67(0.35)$ & 0.12 \\
B & $0.55(0.06)$ & $0.76(0.25)$ & 0.0007 \\
IC & $0.45(0.09)$ & $0.63(0.25)$ & 0.02 \\
Disease duration (years) & & $9(4)^{*}$ & \\
\hline
\end{tabular}

CTR, controls; PAPS, primary antiphospholipid syndrome; IMT, intima media thickness; CC, common carotid; B, carotid bifurcation; IC, internal carotid. ${ }^{*} \mathrm{p}=0.02$ (Mann-Whitney $t$ test). concentration was higher in patients with PAPS than in controls (11.9 (6.2) v 8.2 (3.4) $\mu \mathrm{mol} / \mathrm{l}, \mathrm{p}=0.037$ ).

Overall, IMT was slightly greater at the bifurcation and internal carotid artery in patients with PAPS than in controls (table 2). By chance, our cohort had an equal number of participants below $31(n=10)$ and above $40(n=10)$ years of age. Patients with PAPS aged over 40 had a thicker intima media than patients with PAPS aged less than 31 in all carotid segments considered (table 2).

\section{DISCUSSION}

Atherosclerosis is an important concern in PAPS because it may enhance the cardiovascular burden and contribute further to the morbidity of affected patients, causing, for instance, renal artery stenosis with consequent hypertension, detected in $26 \%$ of patients with APS. ${ }^{13}$ Only two studies have assessed IMT in patients with PAPS.

Bilora et al compared other vascular risk factors in 45 patients with PAPS presenting with deep vein thrombosis with those in an equal number of age and sex matched thrombotic controls similar to the PAPS group. ${ }^{5}$ Having defined an IMT of $>2 \mathrm{~mm}$ as an atherosclerotic plaque, the authors found no difference in the prevalence of plaque at the abdominal aorta and carotid and femoral arteries between patients and controls and concluded that atherosclerosis was not a feature of their PAPS cohort. In contrast, Medina et al found markedly thicker carotid intima media in 28 patients with PAPS than in age and sex matched controls. ${ }^{6}$

However, most of the patients in the latter study ${ }^{6}$ had other conventional risk factors for atherosclerosis, whether in isolation or combination, which might have masked the atherogenic potential of aPL. Of our patients, only 5\% had hypertension and $20 \%$ had hyperlipidaemia compared with the $35 \%$ and $53 \%$, respectively, reported in Medina's cohort. Likewise, 33\% of Bilora's patients had hypertension, 17\% diabetes mellitus, and $29 \%$ hyperlipidaemia. ${ }^{5}$ None of our patients were obese, but $30 \%$ smoked (between 10 and 20 cigarettes a day) compared with $10 \%$ from Medina et $a l^{6}$ and $37 \%$ from Bilora et al. ${ }^{5}$

We also measured plasma $\mathrm{HC}$, and found that the mean concentration was higher in patients with PAPS, although the proportion of participants with HC above the upper limit of normal did not differ. Plasma HC correlates with IMT in patients with PAPS. ${ }^{9}$

Neither of the two studies provided aPL titres of their patients. ${ }^{5}$ IgG aCL were found to independently predict IMT of carotid arteries, highlighting a possible dose effect of aPL on the vessel wall. ${ }^{9}$ The IMT measured by Medina et a ${ }^{6}$ was much higher than in our patients. Racial, genetic, and dietary factors might partly account for these differences. ${ }^{14}$ Moreover, neither of the two studies ${ }^{5}$ accounted for an age effect, whereas we show that patients with PAPS aged $>40$ have greater IMT than controls and than patients with PAPS aged $<30$, although the older patient group had longer disease duration-that is, time from the first thrombotic event to time of the Doppler measurement.

Unfortunately, none of these studies can confirm or refute the premature atherosclerosis hypothesis in patients with PAPS. When compared with healthy controls, patients with PAPS differ for three variables: the presence and persistence of aPL, the occurrence of thrombosis, and continuing oral anticoagulation. Bilora et al correctly employed thrombotic controls, ${ }^{5}$ but with thrombosis of unknown cause, and their duration of anticoagulation might have differed from that of the patients with PAPS. In addition, the authors focused on the relationship between plaque frequency and cumulative positivity in three subgroups of aPL, but not on their titre. We feel that PAPS should be compared with other conditions with persisting occlusive risk, such as the inherited 
thrombophilias. On this group we are performing IMT measurements to match our patients with PAPS for age, sex, occlusive event and, where possible, timing of occlusive event and length of oral anticoagulation. The implication of atherosclerosis in PAPS is of such importance that any study attempting to assess its presence should be as controlled as possible to avoid confounding. Our preliminary results suggest that premature atherosclerosis occurs at least in older patients with PAPS, but this requires validation according to the above-mentioned criteria.

\section{Authors' affiliations}

P R J Ames, K B Sokoll, Academic Department of Rheumatology, Leeds General Infirmary, Great George Street, Leeds LS1 3EX, UK M Weston, Department of Radiology, St James's University Hospital, Beckett Street, Leeds LS9 7TF, UK

A Margarita, V Brancaccio, Angiology and Coagulation Units, Cardarelli Hospital, Via A. Cardarelli, 80100, Naples, Italy

Correspondence to: Dr P R J Ames, Academic Department of Rheumatology, Leeds General Infirmary, Great George Street, Leeds LS1 3EX, UK; paxmes@aol.com

\section{Accepted 9 May 2004}

Published Online First 21 May 2004

\section{REFERENCES}

1 Ames PRJ. Medical perspective. Antiphospholipid antibodies, thrombosis and atherosclerosis in systemic lupus erythematosus. A unifying "membrane stress syndrome" hypothesis. Lupus 1994:3:371-7.

2 Svenungsson E, Jensen-Urstad K, Heimburger M, Silveira A, Hamsten A, de Faire $U$, et al. Risk factors for cardiovascular disease in systemic lupus erythematosus. Circulation 2001;104:1887-93.
3 Roman MJ, Salmon JE, Sobel R, Lockshin MD, Sammaritano L, Schwartz JE, et al. Prevalence and relation to risk factors of carotid atherosclerosis and left ventricular hypertrophy in systemic lupus erythematosus and antiphospholipid antibody syndrome. Am J Cardiol 2001;87:663-6.

4 Manzi S, Selzer F, Sutton-Tyrrell K, Fitzgerald SG, Rairie JE, Tracy RP, et al. Prevalence and risk factors of carotid plaque in women with systemic lupus erythematosus. Arthritis Rheum 1999:42:51-60.

5 Bilora F, Boccioletti V, Girolami B, Zanon E, Armani M, Petrobelli F, et al. Are antiphospholipid antibodies an independent risk factor for atherosclerosis? Clin Appl Thromb Hemost 2002;8:103-13.

6 Medina G, Casaos D, Jara U, Vera-Lastra O, Fuentes M, Barile L, et al. Increased carotid artery intima-media thickness may be associated with stroke in primary antiphospholipid syndrome. Ann Rheum Dis 2003;62:607-10.

7 Nityanand S, Bergmark C, de Faire U, Swedenborg J, Holm G, Lefvert AK. Antibodies against endothelial cells and cardiolipin in young patients with peripheral atherosclerotic disease. J Intern Med 1995;238:437-43.

8 Glueck CJ, Lang JE, Tracy T, Sieve-Smith L, Wang P. Evidence that anticardiolipin antibodies are independent risk factors for atherosclerotic vascular disease. Am J Cardiol 1999;83:1490-4.

9 Ames PRJ, Margarita A, Delgado Alves J, Tommasino C, lannaccone L, Brancaccio V. Anticardiolipin antibody titre and plasma homocysteine level independently predict intima media thickness of carotid arteries in subjects with idiopathic antiphospholipid antibodies. Lupus 2002;11:208-14.

10 Wilson WA, Gharavi AE, Koike T, Lockshin MD, Branch DW, Piette JC, et al. International consensus statement on preliminary classification criteria for definite antiphospholipid syndrome: report of an international workshop. Arthritis Rheum 1999;42:1309-11.

11 Greaves M, Cohen H, Machin SJ, Mackie I. Guidelines on the investigation and management of the antiphospholipid syndrome. Br J Haematol 2000;109:704-15.

12 Burke GL, Evans GW, Riley WA, Sharrett AR, Howard G, Barnes RW, et al. Arterial wall thickness is associated with prevalent cardiovascular disease in middle-aged adults: the Atherosclerosis Risk in Communities (ARIC) Study. Stroke 1995;26:386-91.

13 Sangle SR, D'Cruz DP, Jan W, Karim MY, Khamashta MA, Abbs IC, et al. Renal artery stenosis in the antiphospholipid syndrome and hypertension. Ann Rheum Dis 2003;62:999-1002.

14 Kuller LW. Ethnic differences in atherosclerosis, cardiovascular disease and lipid metabolism. Curr Opin Lipidol 2004;15:109-13. 